\title{
ESPECIE NUEVA DE ANFÍPODO DEL GÉNERO PHOTIS (GAMMARIDEA: PHOTIDAE) DEL ARCHIPIÉLAGO CUBANO
}

\author{
Manuel Ortiz ${ }^{1}$, Carlos Varela ${ }^{2}$ y Rogelio Lalana ${ }^{1}$ \\ ${ }^{1}$ Centro de Investigaciones Marinas, Universidad de La Habana, Calle 16, No. 114 e/ 1ra. y 3ra, Playa, \\ La Habana, Cuba.normano@uh.cu \\ ${ }^{2}$ Acuario Nacional de Cuba (ANC), Calle 1ra \#6002 e/. 60 y 62, Playa, La Habana, Cuba \\ carlosv@acuarionacional.cu
}

\section{RESUMEN}

Se describe una especie nueva de anfípodo del género Photis colectada en las aguas de la Región Oriental del archipiélago cubano. Se presentan las diferencias más notables de dicha especie con las dos más semejantes presentes en el área: Photis sp. F (LeCroy, 2000) y P. sarae Souza-Filho y Serejo, 2010. Es la tercera especie del género registrada en las aguas cubanas.

Palabras clave: Amphipoda, Gammaridea, Photidae, Photis, especie nueva, Cuba.

\section{ABSTRACT}

A new species of amphipod crustacean of the genus Photis collected at the North Eastern waters of the Cuban Archipelago is described. The main differences among this new species and those two most similar recorded in the area (Photis sp. F. LeCroy, 2000 and P. sarae Souza-Filho y Serejo, 2010) are presented. This is the third species of Photis recorded for Cuban waters.

Key word: Amphipoda, Gammaridea, Photidae, Photis, new species, Cuba.

\section{INTRODUCCIÓN}

El género Photis se encuentra representado en el Golfo de México por las siguientes especies: P. longicaudata Bate y Westwood, 1863, P. macromana Mckinney, Kalke y Holland, 1978, P. melanica, McKinney, 1980, P. pugnator Shoemaker, 1945, P. trapherus Thomas y Barnard, 1991, Photis sp. C, Photis sp. D, Photis sp. E y Photis sp. F (LeCroy, 2000). Recientemente, LeCroy et al. (2009) solo menciona las primeras cinco. Las especies de este género que habitan en el Mar Caribe han sido citadas por Ortiz et al. (2007). Este género está representado en el archipiélago cubano, por P. trapherus Thomas y Barnard, 1991, y Photis sp. (Ortiz et al., 2006.)

Recientemente, una nueva especie fue descrita en Brazil, Photis sarae Souza-Filho y Serejo, 2010. La cual está relacionada con P. hawaiensis Barnard, 1955 y con Photis sp. F (Le Croy, 2000). En el presente trabajo se describe una nueva especie de Photis, cercana a Photis sp. F y $P$. sarae, de la costa nororiental de Cuba.

\section{OBJETIVO}

- Describir una especie nueva de anfípodo del género Photis, recolectada en aguas de la Región Oriental del archipiélago cubano.

\section{MATERIALES Y MÉTODOS}

El material estudiado provino de lavado de macroalgas colectadas con el auxilio de SCUBA. Las figuras fueron realizadas con la ayuda de una cámara clara. El sistema de clasificación empleado ha sido el de LeCroy et al. (2009). 


\section{RESULTADOS}

\section{TAXONOMÍA}

Orden Amphipoda Latreille, 1816

Suborden Corophiida Leach, 1814

Familia Photidae Boeck, 1871

Género Photis Kroyer, 1842

Photis lecroyae sp. nov.

Figuras 1-4

Diagnosis. Primera a cuarta coxas desprovistas de largas setas. Esquina anteroventral de la primera coxa armada con tres pequeñas setas. Artejo dos del margen posterior del pereiópodo tres sin hileras de largas setas. Con bordes de estridulación en el borde ventral de las coxas tercera y cuarta. Pereiópodo seis normalmente desarrollado, más corto que la mitad de la longitud total del cuerpo (no tan grande como en P. trapherus Thomas y Barnard, 1991). Margen anteromedial del segundo artejo del segundo gnatópodo presentando un proceso laminar lingüiforme. Pereiópodos del cinco al siete con espina accesoria en el séptimo artejo. Urópodos primero y segundo con una espina marginal en cada rama externa.

Diagnosis. Coxae first till fourth devoid of long setae. Antero ventral corner of first coxa armed with three small setae. Article two of posterior margin of pereopod three not lined with long setae. With stridulating ridges on ventral border of coxae third and fourth. Pereopod sixth normally developed, shorter than half length of body (not as big as in P. trapherus Thomas \& Barnard, 1991) Anteromedial margin of second article on the second gnatopod bearing a linguiform laminar process. Pereopods five to seven with accessory spine on seventh article. First and second uropods with one marginal spine on each outer ramus.

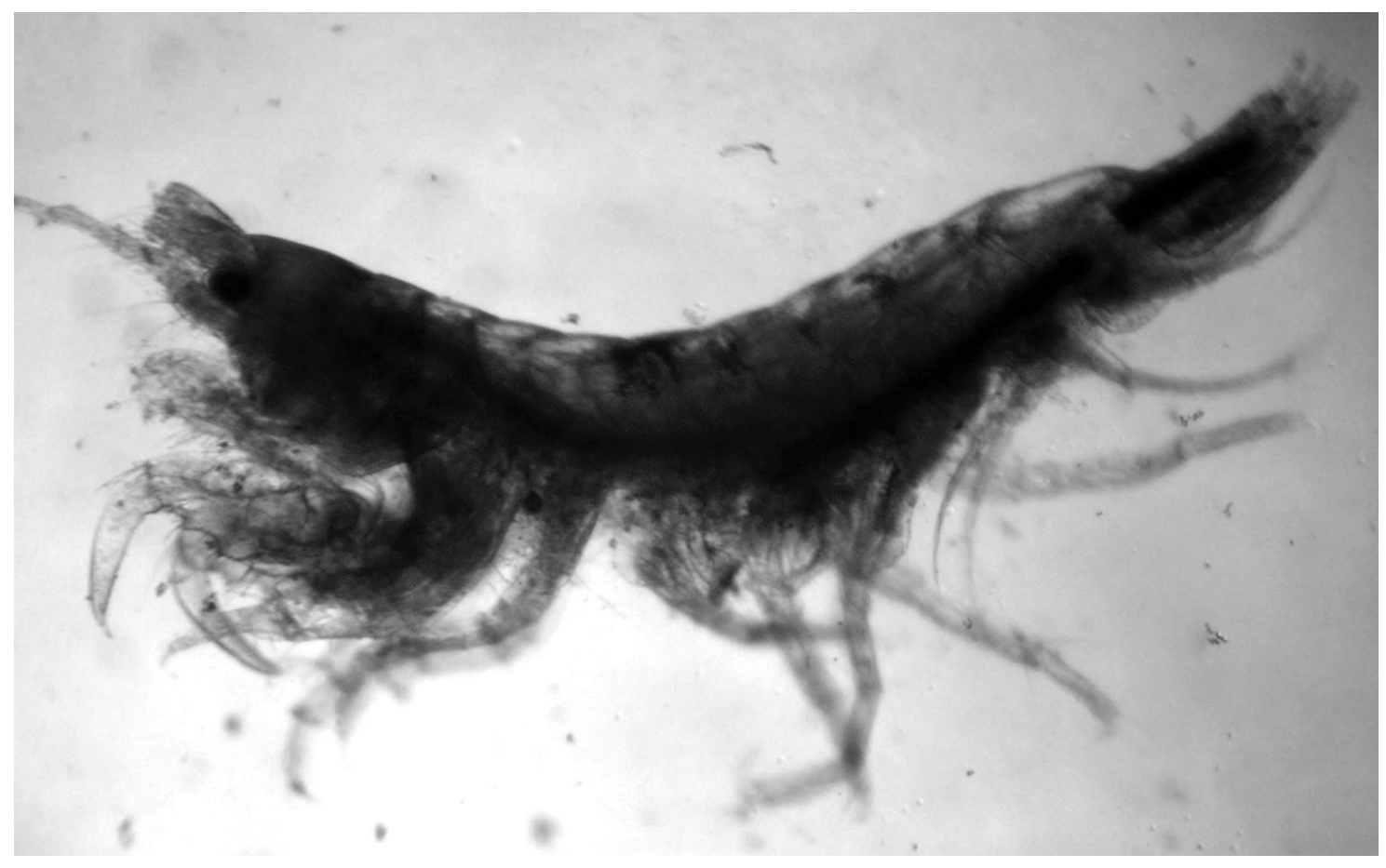

Figura 1. Holotipo, macho. Vista lateral. 

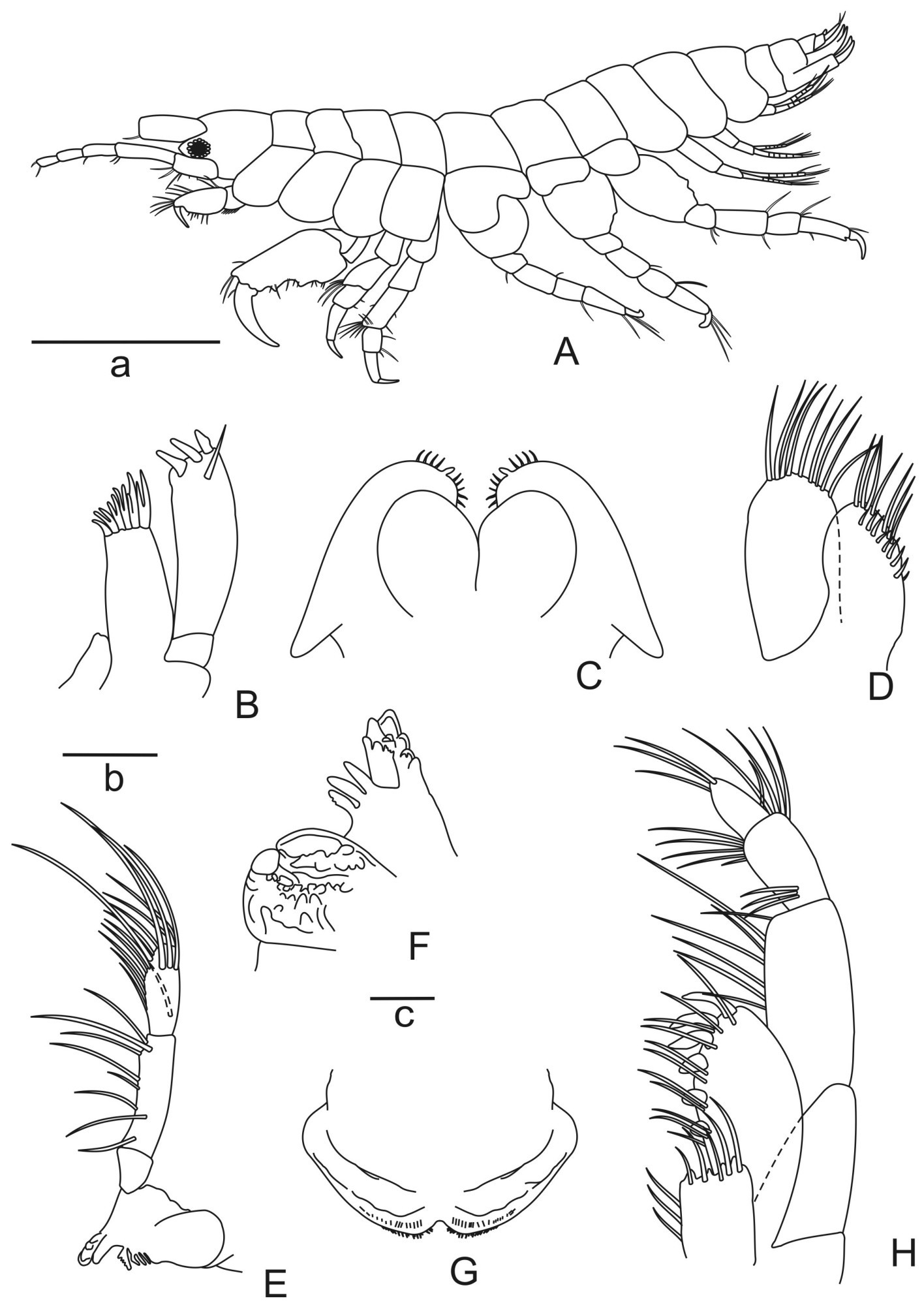

Figura 2. Macho Holotipo. A. Vista lateral (a). B. Maxila 1 (b). C. Labio inferior (b). D. Maxila 2 (b). E. Mandíbula izquierda y palpo (b). F. Mandíbula derecha (c). G. Labio superior (b). H. Maxilípedo (b). Escala. a: $500 \mu \mathrm{m}$; b: $50 \mu \mathrm{m}$ y c: $20 \mu \mathrm{m}$. 
Descripción del holotipo. Lóbulo ocular de la cabeza distalmente subagudo. Ojo bien desarrollado, con numerosos ommatidios (fig. 1). Seno antenal inferior muy retrasado en relación a la inserción de la antena 2 . Antena 1, primer artejo del pedúnculo antenal con una seta en la esquina anteroventral; resto de la antena perdido. Antena 2 con dos largas setas en la esquina posterodistal del artejo del pedúnculo basal; una seta en el segundo y tercer artejo respectivamente; flagelo aparentemente roto (fig. $2 \mathrm{~A}$ ).

Palpo del maxilípedo con cuatro artejos; artejo dos armado con siete setas en el borde interno; artejo tres con tres setas basales y cinco distales en el borde interno, y cuatro en el extremo distal externo; lóbulo externo con seis dientes odontoideos y once setas en su borde interno; lóbulo interno truncado distalmente, armado con cuatro dientes y cinco setas; borde interno cubierto con largas setas (fig. $2 \mathrm{H}$ ).

Extremo distal del lóbulo externo de la maxila 1 con cinco espinas bífidas y una simple. Extremo distal del lóbulo interno desprovisto de setas (fig. 1 B). Lóbulo externo de la maxila 2 con nueve largas setas distales; lóbulo interno con siete setas distales y ocho setas faciales cortas (fig. 2 D). Labio superior redondeado; escindido y cubierto con setas diminutas (fig. 2 G). Lóbulos externos del labio inferior con setas y "procesos digitiformes" en su porción media interior; con lóbulos internos bien desarrollados (fig. 2 C).

Mandíbulas similares entre sí; cinco dientes insicivos; con 1-4 dientes en el extremo distal de la lacinia mobilis; y 3-4 setas accesorias; molar redondeado, bien desarrollado; artejo 3 del palpo más corto que el 2 (figs. 2 E y F).

Epímeros 1-3 redondeados; desprovistos de setas (fig. 3 C). Esquina anteroventral de la coxa 1 casi redondeada de borde entero; seta anteroventral gruesa y truncada, armada con dos setas muy pequeñas.

Artejo 2 del gnatópodo 1 curvado hacia delante, con tres largas setas posteriores y una larga seta anterior; artejos 3 y 4 con una agrupación de largas setas en su porción posterodistal; artejo 5 casi tan largo como el 6, con dos agrupaciones de largas setas posteriores, artejo 6 con el borde de la palma oblicuo, ángulo de la palma pobremente definido, con un grupo de setas largas y cortas, artejo 7 con un diente posterior subdistal (fig. 3 A). Gnatópodo 2 fuerte; artejo 2 desprovisto de setas; margen anteromedial del artejo 3 con procesos laminares; artejo 5 con agrupaciones de largas setas en su región anterior y posterior; borde de la palma del artejo 6 presenta tres procesos, primero y tercero agudos, segundo no prominente; artejo 7 fijando la palma, armado con un diente distal (fig. 3 B).

Pereiópodos 3-7 armados con muy pocas setas. Pereiópodos 3 y 4 muy similares, artejo 5 más corto que el 4 y el 6, armados con una o dos largas setas anterodistales; artejo siete casi tan largo como el artejo 5. Coxas 5 y 6 con lóbulo posterior redondeado (figs. 3 D y E). Artejo 2 del pereiópodo 5 ovoidal; artejo 5 más corto que el 6; artejo 6 armado con una espina distal fuerte; artejo siete muy corto.

Pereiópodo 6 de talla normal; artejo 2 formando un lóbulo posterior redondeado; artejo 4 tan largo como el artejo 5; artejo 6 más largo que el 5. Pereiópodo 7 un poco mayor que el 6; artejo 2 estrechándose distalmente; artejo 6 más largo que el 5 (figs. 4 A a C). 

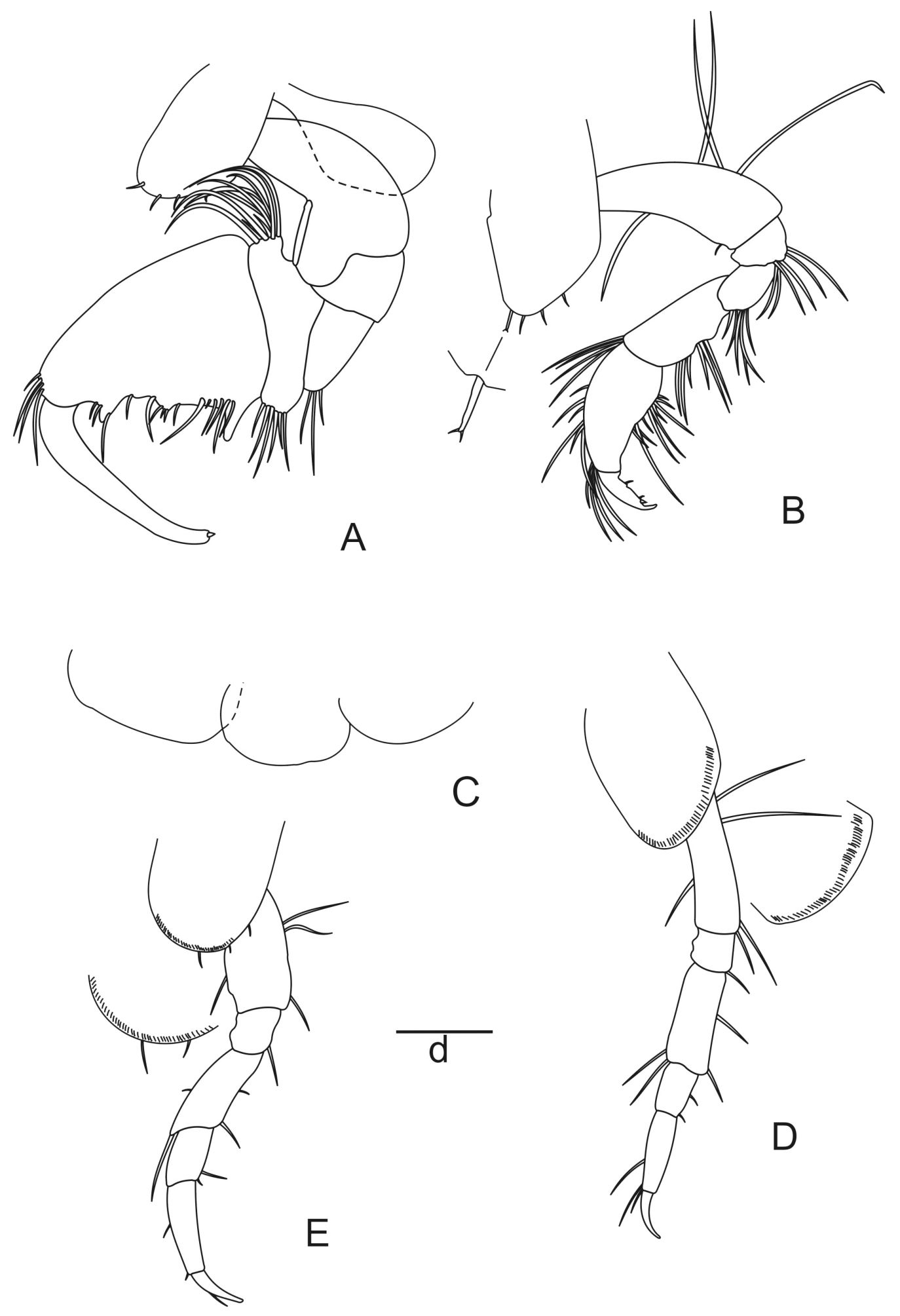

Figura 3. Macho Holotipo. A. Gnatópodo 1 (d). B. Gnatópodo 2 (d). C. Coxas 1-3 (d). D. Pata 3 (d). E. Pata 4 (d). Escala. d: 100 m. 


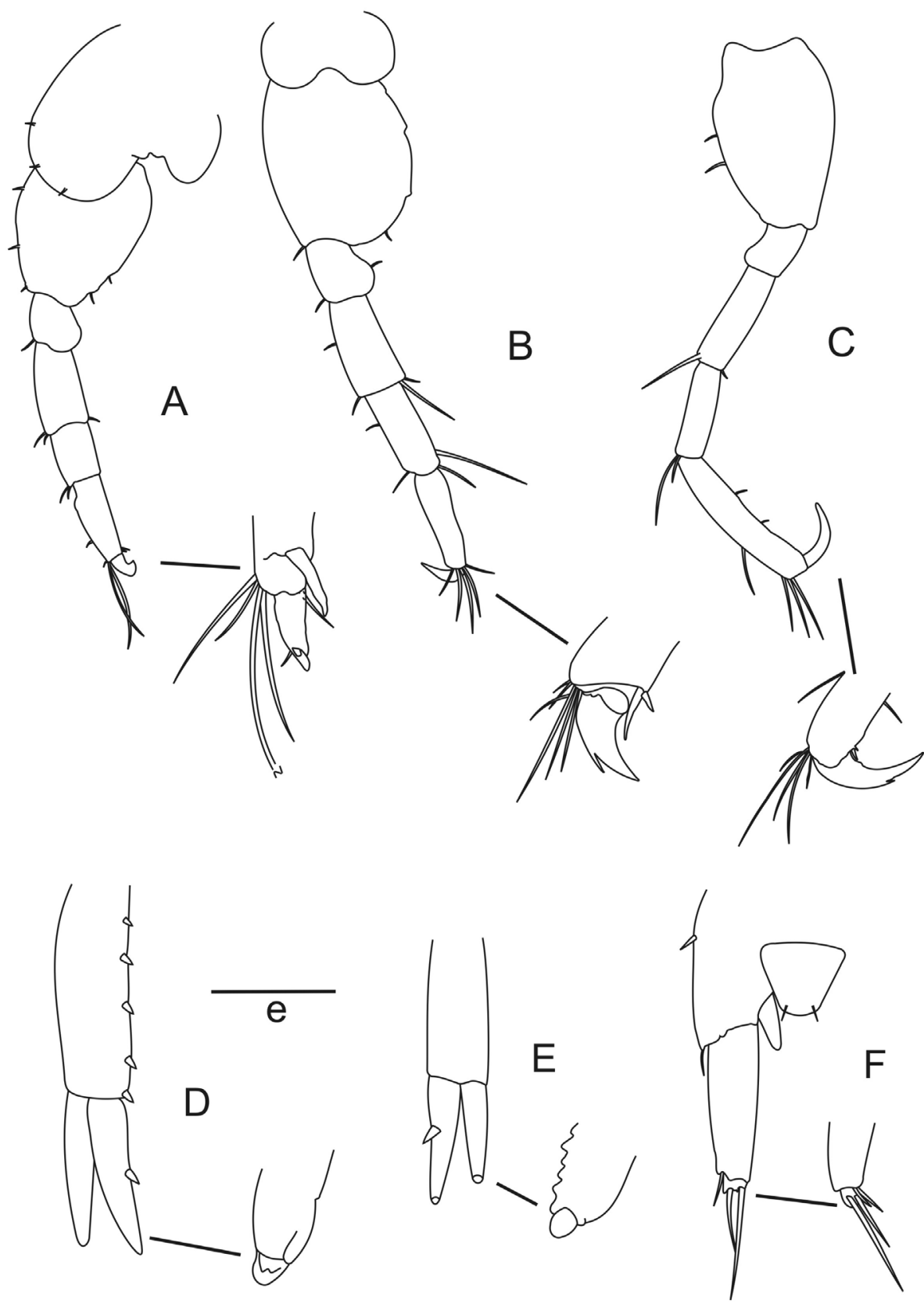

Figura 4. Macho Holotipo. A. Pata 5 (d). B. Pata 6 (d). C. Pata 7 (d). D. Urópodo 1 (e). E. Urópodo 2 (e). F. Urópodo 3 con Telson (e). Escala. e: $50 \mu \mathrm{m}$.

Pedúnculo del urópodo 1 armado con 5 dientes; rama interna con un diente; rama externa desnuda (fig. 4 D). Pedúnculo del urópodo 2 desprovisto de dientes; rama externa más larga que la interna; rama externa armada con un diente (fig. 4 E). Rama interna del urópodo 3 un tercio de la longitud de la externa; punta del primer artejo de la rama externa con dos setas externas cortas; segundo artejo de la rama externa armado con una seta larga y otra corta. Telson trapezoidal, armado con una seta en cada ángulo posterodistal (fig. 4 F). 
Tipos. Holotipo, macho; $1.8 \mathrm{~mm}$; Puerto Padre, costa norte de la provincia de Las Tunas; 27. VIII. 2009; 8 metros de profundidad. El material se encuentra depositado en la colección de invertebrados de la Colección Natural Marina del Acuario Nacional de Cuba. ANC. 07. 1. 2. 012.

Hembra. Desconocida.

Distribución. Solo es conocida para la localidad tipo.

Etimologia. Photis lecroyae sp. nov. es nombrada en honor a la Dra. Sarah E. LeCroy, por su gran contribución a la taxonomía de Amphipoda.

Comentarios. De las especies pertenecientes al género Photis conocidas en el Atlántico Occidental Tropical, $P$. lecroyae sp. nov. es más afín a Photis sp. F (LeCroy, 2000) y P. sarae Souza-Filho y Serejo, 2010, por tener órgano estridulante en el margen ventral de las coxas 3 y 4, gnatópodo 2 con un proceso anteromesial setoso; pereiópodo 6 normal, y espina accesoria en el artejo 7 del pereiópodo 7. Sin embargo, P. lecroyae sp. nov. difiere por tener solo una seta en la esquina distal del primer artejo del pedúnculo de la antena 1; lóbulo interno de la maxila 1 con extremo distal truncado; labio inferior con procesos digitiformes en la porción media del lóbulo externo; lóbulo externo del maxilípedo armado con seis dientes odontoideos largos y cortos; borde posterior del artejo 2 del gnatópodo 2 desprovisto de setas; artejo 2 estrecho en los pereiópodos 5 y 6 ; telson armado solo con una seta en cada esquina posterior; rama interna del urópodo 3 desprovista de setas terminales; una seta gruesa y truncada en la esquina anteroventral de la coxa 1; artejo 2 del palpo mandibular largo, artejo 7 del gnatópodo 1 armado con un diente subdistal y artejo 7 del gnatópodo 2 presenta un diente distal.

\section{LITERATURA CITADA}

LeCroy, S. 2000. An illustrated identification guide to the nearshore marine and estuarine gammaridean Amphipoda of Florida. 1 Families Gammaridae, Hadziidae, Isaeidae, Melitidae and Oedicerotidae. Florida Department of Environmental Protection Division of Resources Assessment and Management Bureau Laboratory, Stone Road, Tallahassee, Florida. 195 pp.

LeCroy, S., R. Gasca, I. Winfield, M. Ortiz y E. Escobar-Briones. 2009. Chapter 54. Amphipoda (Crustacea) of the Gulf of Mexico. In: Felder, D. L. y D. R. Camp (editors) Gulf of Mexico, Origin, Waters, and Biota, Vol. 1, Biodiversity. Texas A\&M University Press, U. S. A. 941-972 pp.

Ortiz, M., R. Lalana y C. Varela. 2006. Crustáceos no decápodos. Filo Arthropoda, Sub-filo Crustacea. Lista de especies registradas en Cuba (Octubre de 2006). En: Claro, R. (editor), La Biodiversidad Marina de Cuba, Instituto de Oceanología, Ministerio de Ciencias, Tecnología y Medio Ambiente. CD-ROM.

Ortiz, M., A. Martín y Y. Díaz. 2007. Lista y referencias de los crustáceos anfípodos (Amphipoda, Gammaridea) del Atlántico Occidental Tropical. Revista de Biología Tropical 55 (2): 479-498.

Souza-Filho, J. F. y C. S. Serejo. 2010. Two new species of the family Photidae (Amphipoda, Corophiidea, Photidae) from Brazilian waters, with description of Rocasphotis gen. nov. Journal of Natural History 44 (9): 559-577. 